\title{
Non-toxic agarose/gelatin-based microencapsulation system containing gallic acid for antifungal application
}

\author{
P.-L. LAM ${ }^{1}$, R. GAMBARI ${ }^{2}$, S.H.-L. KOK ${ }^{3}$, K.-H. LAM ${ }^{1}$, J.C.-O. TANG ${ }^{1}$, \\ Z.-X. BIAN ${ }^{4}$, K.K.-H. LEE $^{3}$ and C.-H.CHUI ${ }^{1,4}$ \\ ${ }^{1}$ State Key Laboratory of Chirosciences, Department of Applied Biology and Chemical Technology, \\ The Hong Kong Polytechnic University, Hong Kong, SAR, P.R. China; ${ }^{2}$ Centre of Biotechnology, \\ Department of Life Sciences and Biotechnology, University of Ferrara, Ferrara, Italy; ${ }^{3}$ Ministry of Education (MOE) \\ Key Laboratory for Regenerative Medicine, School of Biomedical Sciences, Chinese University of Hong Kong; \\ ${ }^{4}$ Clinical Division, School of Chinese Medicine, Hong Kong Baptist University, Hong Kong, SAR, P.R. China
}

Received June 12, 2014; Accepted November 10, 2014

DOI: $10.3892 / \mathrm{ijmm} .2014 .2027$

\begin{abstract}
Aspergillus niger (A. niger) is a common species of Aspergillus molds. Cutaneous aspergillosis usually occurs in skin sites near intravenous injection and approximately $6 \%$ of cutaneous aspergillosis cases which do not involve burn or $\mathrm{HIV}$-infected patients are caused by $A$. niger. Biomaterials and biopharmaceuticals produced from microparticle-based drug delivery systems have received much attention as microencapsulated drugs offer an improvement in therapeutic efficacy due to better human absorption. The frequently used crosslinker, glutaraldehyde, in gelatin-based microencapsulation systems is considered harmful to human beings. In order to tackle the potential risks, agarose has become an alternative polymer to be used with gelatin as wall matrix materials of microcapsules. In the present study, we report the eco-friendly use of an agarose/gelatin-based microencapsulation system to enhance the antifungal activity of gallic acid and reduce its potential cytotoxic effects towards human skin keratinocytes. We used optimal parameter combinations, such as an agarose/gelatin ratio of 1:1, a polymer/oil ratio of 1:60, a surfactant volume of $1 \% \mathrm{w} / \mathrm{w}$ and a stirring speed of $900 \mathrm{rpm}$. The minimum inhibitory concentration of microencapsulated gallic acid $(62.5 \mu \mathrm{g} / \mathrm{ml})$ was significantly improved when compared with
\end{abstract}

Correspondence to: Professor K.K.-H Lee, Ministry of Education (MOE) Key Laboratory for Regenerative Medicine, School of Biomedical Sciences, Chinese University of Hong Kong, Tai Po Road, Shatin, Hong Kong, SAR, P.R. China

E-mail:kaholee@cuhk.edu.hk

Dr C.-H. Chui, Clinical Division, School of Chinese Medicine, Hong Kong Baptist University, 11 Yuk Choi Road, Hung Hom, Hong Kong, SAR, P.R. China

E-mail: chchui@hkbu.edu.hk

Keywords: Aspergillus niger, agarose, gelatin, gallic acid, microcapsules that of the original drug $(>750 \mu \mathrm{g} / \mathrm{ml})$. The anti-A. niger activity of gallic acid -containing microcapsules was much stronger than that of the original drug. Following $48 \mathrm{~h}$ of treatment, skin cell survival was approximately $90 \%$ with agarose/gelatin microcapsules containing gallic acid, whereas cell viability was only $25-35 \%$ with free gallic acid. Our results demonstrate that agarose/gelatin-based microcapsules containing gallic acid may prove to be helpful in the treatment of $A$. niger-induced skin infections near intravenous injection sites.

\section{Introduction}

The Aspergillus species includes common saprophytic molds found worldwide (1). Skin diseases usually induced by a cutaneous manifestation of disseminated infection with Aspergillus fungi are known as cutaneous aspergillosis $(2,3)$. Among the Aspergillus molds, Aspergillus niger (A. niger) is an abundant species of Aspergillus found in the environment. A. niger is a filamentous ascomycete fungus that has the potential to cause human diseases (4). Cutaneous aspergillosis usually occurs in the infected skin sites at or near intravenous injection sites and as for the cases of cutaneous aspergillosis which do not involve burn or HIV-infected patients, A. niger is responsible for approximately $6 \%$ of these cases (2). Recently, the concern for the safe use of biomaterials has increased worldwide. Microparticle-based drug delivery is under consideration as it promotes controlled drug release to improve the therapeutic performance of original drugs through human absorption (5). The microparticle system extends the drug circulation time in the body and improves the drug concentration in target tissues. This increases the therapeutic efficacy and reduces the frequency of drug administration (6).

Gelatin, as a biodegradable polymer, requires the use of the crosslinker, glutaraldehyde, to improve the strength of microcapsules (7). Since gelatin rapidly dissolves under aqueous conditions, its application in controlled drug delivery systems is considered difficult without the use of crosslinkers (8). Glutaraldhyde can promote an insoluble network within the wall matrix of microcapsules to reduce dissolution and 
premature drug release (9). Glutaraldehyde is commonly used for medical, scientific, industrial and cosmetic applications. However, glutaraldehyde is considered a toxic and hazardous agent which can induce various adverse health effects on the human body, including allergic contact dermatitis, eye irritations and respiratory sensitizations $(10,11)$. Previous studies have reported the potential health effects of glutaraldehyde exposure to human beings. Cross-sectional studies have demonstrated an increase in symptoms, such as skin sensitizations among medical workers when they were exposed to glutaraldehyde $(12,13)$. In vivo animal studies have also investigated the risks of glutaraldehyde exposure. Both female guinea pigs and mice had skin sensitizations with $0.3-3.0 \%$ of glutaraldehyde and dose-dependent contact hypersensitivity (14). Recently, we demonstrated the use of an agar/gelatin-based microencapsulation system for the delivery of Phyllanthus urinaria for antifungal application (15). Treatment with an agar/gelatin-based system containing Phyllanthus urinaria led to a slight improvement in antifungal activity due to a comparatively weak strength of agar gel in the microencapsulation system (15). In order to enhance the antifungal effects, agarose may be an alternative polymer to be combined with gelatin as wall matrix materials of microcapsules to enhance the potential antifungal effects of the entrapped antifungal agent and prevent the harmful effects of using the hazardous crosslinker. Agarose, as a linear polysaccharide based on the 3,6-anhydro$\alpha$-L-galactopyranose unit, is originated from red seaweed. Agarose is natural, biocompatible and biodegradable and it has been widely used in food, cosmetic and pharmaceutical applications (16-18). The major difference between agarose and agar is that agar is composed of a mixture of agarose and agaropectin. Agar consists of agaropectin which contains a heterogeneous mixture of smaller molecules that contribute to weaker mechanical properties (16). The gel formed by agarose is relatively stronger than the agar gel at the same concentration (19). Sakai et al (20) reported the use of a cell-adhesive agarose-gelatin conjugate (Aga-Ge) gel membrane for the enhancement of adherent cell growth. Agarose can be covalently crosslinked with gelatin at a lower temperature to form the gel networks (20).

Gallic acid (3,4,5-trihydroxybenzoic acid) is originated from the plant kingdom, commonly found in phenolic materials, such as tannins, flavonoids and lignin (21). Gallic acid has been reported to exert a number of therapeutic effects, including antimicrobial $(22,23)$, antioxidant (24) and anticancer $(25,26)$ activities. However, the single use of gallic acid is considered harmful and toxic to human beings. Gallic acid can induce the death of endothelial cells (27). Subchronic toxicity symptoms, including extramedullary hematopoiesis, hemosiderin deposition and congestion in the spleens of gallic acid-treated rats have also been reported (28). Gallic acid induces cytotoxic effects towards mouse sertoli cells (21). Recently, we reported the use of an agar/gelatin-based microencapsulation system to deliver gallic acid for hepatoprotection in order to treat liver damage due to an overdose administration of acetaminophen (5). In this study, gallic acid was used as an antifungal agent which was entrapped into an agarose/gelatin-based microencapsulation system so as to enhance its antifungal effects against $A$. niger and reduce its cytotoxicity towards human skin keratinocytes.

\section{Materials and methods}

Materials. Agarose (type VII-A; gel point, $26 \pm 2{ }^{\circ} \mathrm{C}$ at $1.5 \%$ gel; melting point, $\leq 65.5^{\circ} \mathrm{C}$ ) and gelatin (derived from porcine skin, type A) were purchased from Sigma-Aldrich, Steinheim, Germany. Gallic acid (melting point, $>200^{\circ} \mathrm{C}$ ) was kindly provided by the China National Institute for the Control of Pharmaceutical and Biological Products, Beijing, China. Pure olive oil was obtained from Easy Creation Asia Ltd., Hong Kong, China. All the chemical reagents were supplied from Sigma-Aldrich.

Preparation of microcapsules. The method for microcapsule formation was as previously reported with slight modifications $(5,15)$. Agarose and gelatin were hydrated in deionized water and mixed well together at $70-80^{\circ} \mathrm{C}$. Gallic acid was titrated to $\mathrm{pH} 7$ using $1 \%$ of $\mathrm{NaOH}$. The titrated drug was poured into the agarose-gelatin mixture and continuously mixed for $10 \mathrm{~min}$. Pure olive oil with $1 \% \mathrm{v} / \mathrm{v}$ Span 80 as a surfactant was added to the agarose-gelatin-drug mixture followed by continuous stirring to form the water in oil emulsion. The emulsion was then placed in a homogenizer (BioSpec Products, Bartlesville, OK, USA) to form the homogeneous emulsion. The emulsion was allowed to cool down to $<25^{\circ} \mathrm{C}$ and continuously stirred for $3 \mathrm{~h}$. The precipitates were washed with acetone. The resulting microcapsules were suction-filtered and stored in desiccators.

Determination of drug entrapment efficiency. Fifty milligrams of drug-containing microcapsules were dissolved in $100 \mathrm{ml}$ of deionized water by heat. The samples were then filtered. The gallic acid content was analyzed by measuring the absorbance at $260 \mathrm{~nm}$ ( $\lambda_{\max }$ of drugs in deionized water) after suitable dilution using an UV/VIS spectrophotometer (Lambda 18; Perkin Elmer, Waltham, MA, USA). Each sample was analyzed in triplicate in order to obtain the mean entrapment efficiency. The drug entrapment efficiency was calculated using the following equation, as previously described (5):

Drug entrapment efficiency $=\frac{\text { Amount of calculated drug }}{\text { Amount of theoretical drug }} \times 100 \%$

Surface morphology and particle size. Microcapsules were attached to the SEM stub using a twisted adhesive tape. The test samples were gold-coated prior to performing scanning electron microscopy (SEM) observation. The surface morphology of the drug-containing microcapsules was observed using a scanning electron microscope (JSM-6490LV; JEOL, Blackwood, NJ, USA). The particle size of the microcapsules was evaluated according to the captured SEM images. A total number of 500 microcapsules was counted manually, as previously described $(5,7,15)$.

Fourier transform infrared spectroscopy (FTIR) anlaysis. In FTIR analysis, potassium bromide (KBr) was used as it has no pronounced absorption peaks over the mid-infrared band. In this section, $2 \mathrm{mg}$ of samples were pelleted with $200 \mathrm{mg}$ of $\mathrm{KBr}$ through the vacuum process. The chemical compositions of the developed microcapsules were examined using a 


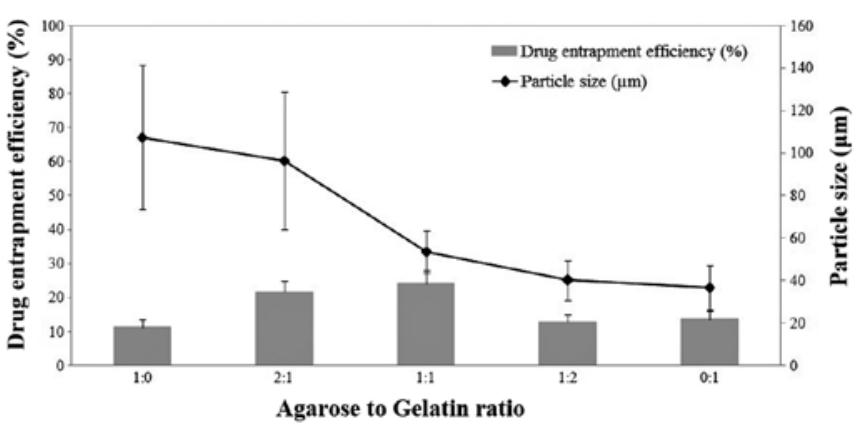

Figure 1. Effect of polymer ratio on the drug entrapment efficiency and particle size of microcapsules.

Spectrum 100 Series FT-IR Spectrometer (Perkin Elmer), as previously described $(5,7,15)$.

In vitro release study. For the in vitro release model of microcapsules containing gallic acid, a given amount of microcapsules was firstly suspended in $1,000 \mathrm{ml}$ phosphate solution ( $\mathrm{pH}$ 7.4) medium for $96 \mathrm{~h}$ and incubated at $100 \mathrm{rpm}$ at room temperature. Aliquots were taken at desired time intervals. The drawn sample was filtered using filter paper and the residue was returned to the suspension medium. The clear filtrate of the drug-containing microcapsules was analyzed using a UV/VIS spectrophotometer (Perkin Elmer) for the determination of drug content, as previously described $(5,7,15)$.

Determination of minimum inhibitory concentrations (MICs). Gallic acid was titrated to $\mathrm{pH} 7$ to avoid too much aciditiy initially and improve the drug solubility. The conversion of gallic acid to a compound with the ketone group was believed to increase the antibacterial activity of the original drug. MICs of gallic acidcontaining microcapsules and gallic acid were determined by the LB broth dilution method. A. niger in a concentration of $1 \times 10^{3}$ cells $/ \mathrm{ml}$ was added to the test tubes. Various concentrations of gallic acid-containing microcapsules and gallic acid were added from a starting concentration of $1,000 \mu \mathrm{g} / \mathrm{ml}$ and were serially diluted. Terbinafine was employed as the positive control, while blank microcapsules (without drugs) were used as a negative control. Afterwards, they were incubated at $25^{\circ} \mathrm{C}$ for $48 \mathrm{~h}$. The minimum concentrations of gallic acid-containing microcapsules and gallic acid that caused a complete growth inhibition towards microorganisms were recorded as MICs (15).

Antifungal study. A. niger in a concentration of $1 \times 10^{3}$ cells $/ \mathrm{ml}$ was placed on agar plates. Filter papers were cut in a circular shape $1 \mathrm{~cm}$ in diameter. Paper stripes containing microencapsulated gallic acid and the free gallic acid at various concentrations were placed on the surface of the nutrient agar. Terbinafine $(5 \mu \mathrm{g})$ was used as a positive control, while blank microcapsules without drugs were applied as a negative control. The plates were incubated in an incubator at $25^{\circ} \mathrm{C}$ for 2 days and the growth inhibitory effects of $A$. niger on the agar plates were recorded, as previously described (15).

Cytotoxicity assay. Human cells, including $\mathrm{HaCaT}$ skin keratinocytes were removed from $75 \mathrm{ml}$ sterile cell culture flasks with trypsin and neutralized with fetal bovine serum. After

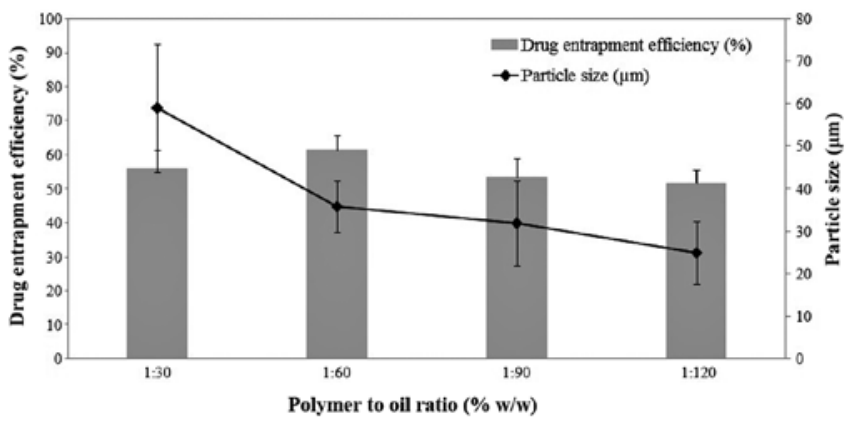

Figure 2. Effect of polymer to oil ratio on the drug entrapment efficiency and particle size of microcapsules.

washing with phosphate-buffered saline and centrifugation, the skin cells were re-suspended in complete cell culture medium at a concentration of approximately $1 \times 10^{5}$ cells $/ \mathrm{ml}$ and counted manually using a haematocytometer under an inverted microscope. Human cells seeded in the 24 wells microtitre plates for $24 \mathrm{~h}$ were prepared for the screening of our microcapsules. Microcapsules contaning 125 and $62.5 \mu \mathrm{g}$ of gallic acid, blank microcapsules without drugs and 125 and $62.5 \mu \mathrm{g}$ of free gallic acid were added and incubated with the cells for a further $48 \mathrm{~h}$. Doxorubicin was used as a positive reference. The assessment of cytotoxicity was conducted using the sulforhodamine B protein staining method. Skin cells were fixed with trichloroacetic acid, washed with deionized water and stained with sulforhodamine B. Subsequently, the cells were washed again with acetic acid and the stained cells were dissolved in $100 \mathrm{ml}$ unbuffered Tris-base. Finally, optical images of the skin keratinocytes were captured (Canon, Japan), as previously described $(7,29)$.

Statistical analyses. The values are presented as the means \pm standard deviation (SD), and statistical analyses were performed using a student t-test. The value of $\mathrm{P}<0.05$ was considered to indicate a statistically significant difference.

\section{Results and Discussion}

Optimization of agarose/gelatin-based microcapsules containing gallic acid

Effect of polymer ratio. Microcapsule properties may be influenced by the polymer ratio during the formation process. It is vital for the determination of a suitable ratio between two wall polymeric materials (agarose and gelatin) as this may affect the effective entrapment of the core drug into the wall matrix of microcapsules. As shown in Fig. 1, the drug entrapment efficiency of microcapsules containing gallic acid achieved the highest level when the agarose to gelatin ratio was 1:1. It was also observed that the particle size of the microcapsules containing gallic acid was generally reduced with an increase in the gelatin ratio. Therefore, the agarose to gelatin ratio of $1: 1$ was determined to provide better physical properties for the formed microcapsules, with the highest drug entrapment efficiency and acceptable particle size, and this polymer ratio was used for the microcapsule synthesis for the remaining parameters.

Effect of polymer to oil ratio. The ratio of polymer to oil also determined the drug entrapment efficiency and particle size of the developed microcapsules. As shown in Fig. 2, microcapsules 




Figure 3. Effect of surfactant volume on the drug entrapment efficiency and particle size of microcapsules.

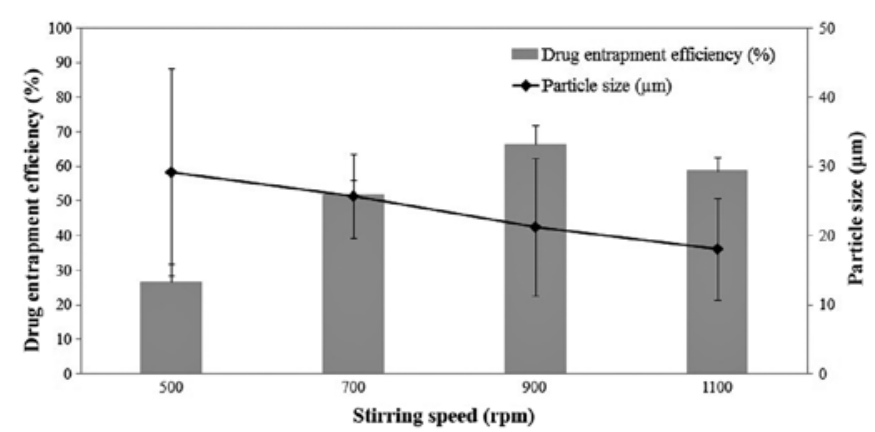

Figure 4. Effect of stirring speed on the drug entrapment efficiency and particle size of microcapsules.

containing gallic acid obtained the maximal drug entrapment efficiency in the polymer to oil ratio of 1:60. Generally, the particle size of the microcapsules containing gallic acid was decreased with the increasing oil ratio. The results may be associated with the fact that the accumulation of microparticles easily occurs in an inadequate oil volume, leading to a larger size of formed microcapsules. However, the water in oil (w/o) emulsion may be processed without difficulties in stirring during the microcapsule production. The microparticles may be easily formed with a better separation as an increase in oil volume at the interface tends to break down the w/o emulsion into smaller micro-droplets (5). Therefore, the selected ratio of polymer to oil was 1:60 in order to produce the microcapsules with higher drug entrapment and satisfactory particle size.
Effect of surfactant volume. The surfactant volume may also result in different drug entrapment efficiencies and particle sizes of microcapsules. Fig. 3 illustrates that the drug entrapment efficiency of the microcapsules containing gallic acid reached the highest level at $1 \% \mathrm{w} / \mathrm{w}$ of the surfactant volume and then decreased. The particle size of the microcapsules decreased when the surfactant volume was increased. This may be associated with a reduction in surface tension and force in the interface between the water phase and oil phase if the surfactant concentration was increased. The better stabilization of emulsified droplets with an increased surfactant input may avoid the aggregation and accumulation of small droplets during the microcapsule formation process (5). The surfactant volume was, consequently, selected as $1 \% \mathrm{v} / \mathrm{v}$ for producing the microcapsules with desirable drug entrapment efficiency and a suitable particle size.

Effect of stirring speed. The stirring speed also became a processing parameter that may differentiate the drug entrapment efficiency and particle size of the formed microcapsules. As shown in Fig. 4, it was observed that the drug entrapment efficiency of the microcapsules containing gallic acid generally increased from the stirring speed of $500 \mathrm{rpm}$ and then reached the maximal level at $900 \mathrm{rpm}$. The particle size of the microcapsules decreased with the increment in stirring speed. This may be due to the fact that a faster stirring speed may break the w/o emulsion into smaller particle droplets and prohibit the smaller microcapsule from agglomerating during the microcapsule manufacturing process. However, a slower stirring speed may induce the cohesion of microparticles which accumulates to larger particles (5). Therefore, the selected stirring speed was $900 \mathrm{rpm}$ so as to develop the microcapsules with the highest drug entrapment and acceptable particle size.

Drug entrapment efficiency, particle size and surface morphology of optimal microcapsules. The optimal mean drug entrapment efficiency of the microcapsules containing gallic acid was estimated to be $61.77 \pm 5.07 \%$. The mean particle size of the gallic acid-loaded microcapsules was $22.73 \pm 6.43 \mu \mathrm{m}$. Fig. 5 illustrates the SEM image of the gallic acid-containing microcapsules. It was observed that they were approximately spherical in shape with a certain level of agglomerations.

FTIR analysis of microcapsules. In FTIR analysis, the diffuse reflectance measurement mode was applied in the range of wavelengths from 4,000 to $450 \mathrm{~cm}^{-1}$ in wave numbers. The
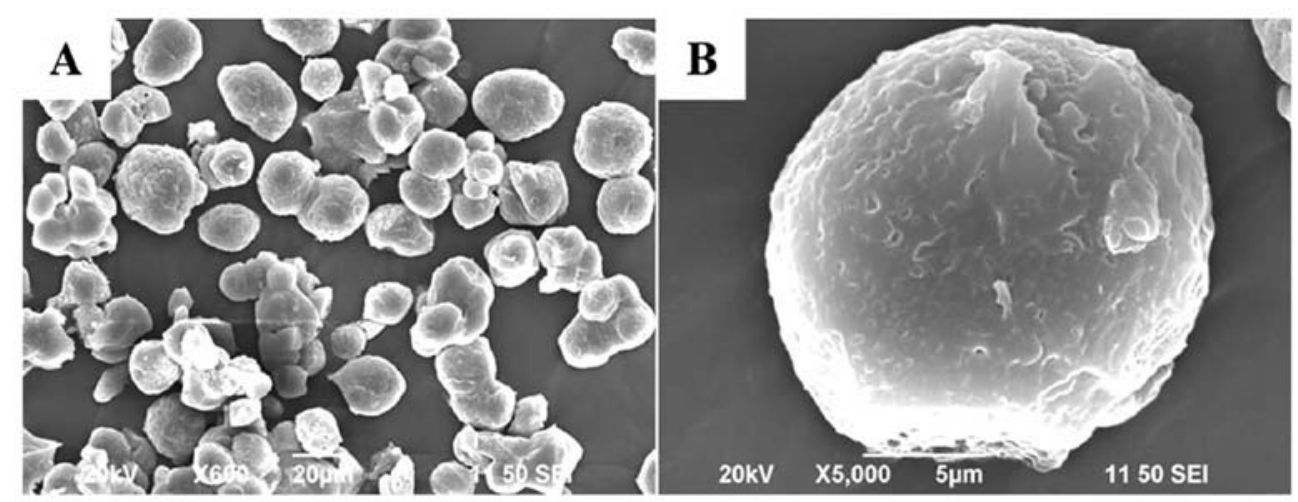

Figure 5. Scanning electron microscopy (SEM) images of agarose/gelatin-based microcapsules containing gallic acid at (A) x600 and (B) x5,000 magnification. 
Table I. MICs of agarose/gelatin-based microcapsules containing gallic acid.

\begin{tabular}{lcccc}
\hline & & MIC $(\mu \mathrm{g} / \mathrm{ml})$ & \\
\cline { 2 - 5 } & $\begin{array}{c}\text { Positive control } \\
\text { (Terbinafine) }\end{array}$ & Gallic acid & $\begin{array}{c}\text { Microcapsules containing } \\
\text { gallic acid }\end{array}$ & Blank microcapsules \\
\hline A. niger & $1.25 \mu \mathrm{g} / \mathrm{ml}$ & $>750 \mu \mathrm{g} / \mathrm{ml}$ & $62.5 \mu \mathrm{g} / \mathrm{ml}$ & Nil \\
\hline
\end{tabular}

MIC, minimum inhibitory concentrations; A. niger, Aspergillus niger.

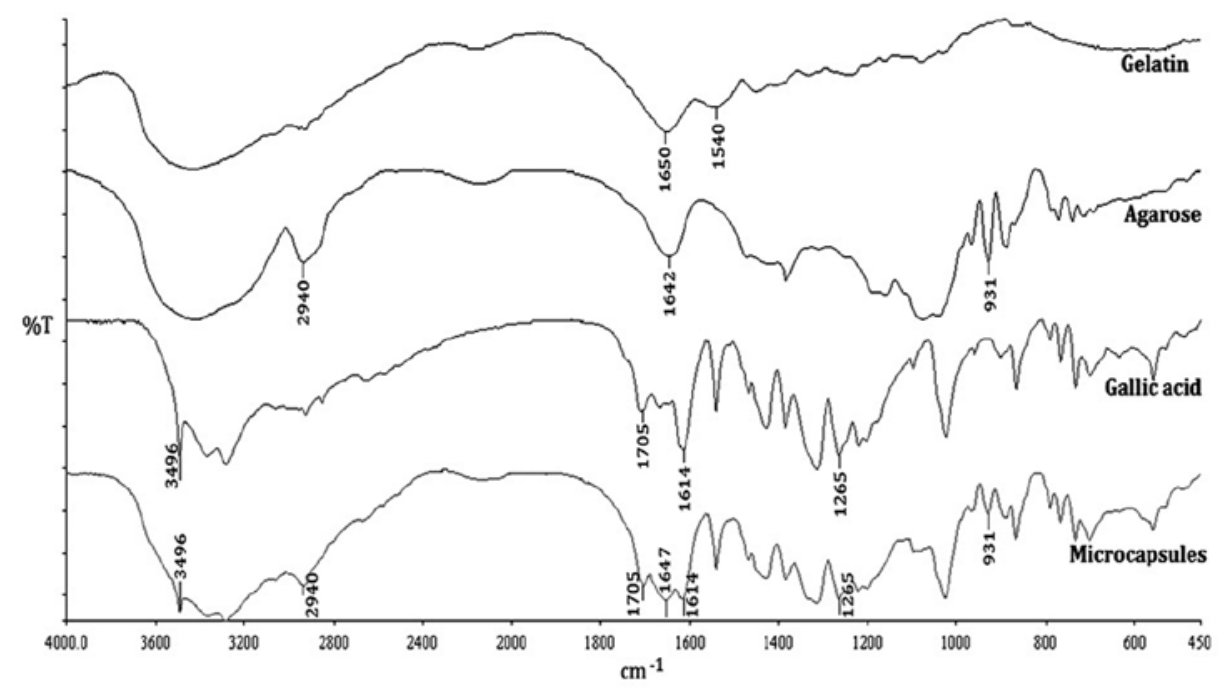

Figure 6. Fourier transform infrared spectroscopy (FTIR) spectra of agarose/gelatin-based microcapsules containing gallic acid.

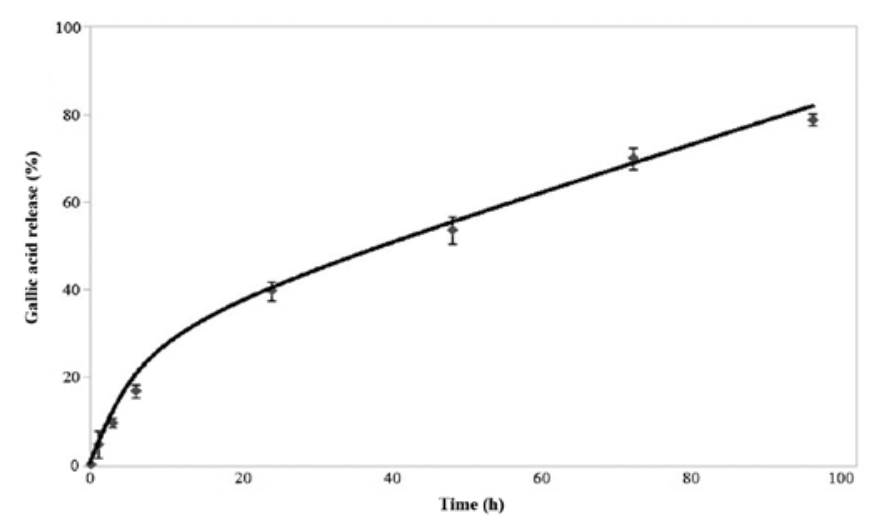

Figure 7. Release profile of agarose/gelatin-based microcapsules containing gallic acid.

infrared absorption spectra of the agarose/gelatin microcapsules containing gallic acid are shown in Fig. 6. Significant peaks were observed in the agarose spectrum at 2,940,1,642 and $931 \mathrm{~cm}^{-1}$ that were related to $\mathrm{C}-\mathrm{H}$ stretching, bonded $\mathrm{H}-\mathrm{O}-\mathrm{H}$ and 3,6-anhydrogalactose linkage, respectively (30). Gelatin showed the characteristic amide absorption bands at 1,656 and $1,540 \mathrm{~cm}^{-1}$ that referred to $\mathrm{N}-\mathrm{H}$ stretching (5). Gallic acid exhibited the characteristic peaks at $3,496 \mathrm{~cm}^{-1}$ (O-H group), $1,705 \mathrm{~cm}^{-1}$ (carboxylic acids), $1,614 \mathrm{~cm}^{-1}$ (C=C stretching) and $1,265 \mathrm{~cm}^{-1}(\mathrm{C}=\mathrm{O}$ stretching $)(5)$. The spectrum of the microcapsules containing gallic acid had the characteristic peaks of gallic acid, agarose and gelatin. The absorbances at 2,940 and $931 \mathrm{~cm}^{-1}$ displayed the $\mathrm{C}-\mathrm{H}$ stretching and 3,6-anhydroglactose linkage from agarose, respectively. The peak at $1,647 \mathrm{~cm}^{-1}$ revealed the presence of bonded $\mathrm{H}-\mathrm{O}-\mathrm{H}$ from agarose and $\mathrm{N}-\mathrm{H}$ stretching from gelatin in the formed microcapsules. The characteristic peaks for gallic acid appeared in $3,496 \mathrm{~cm}^{-1}$ (O-H group), $1,705 \mathrm{~cm}^{-1}$ (carboxylic acids), $1,614 \mathrm{~cm}^{-1}$ ( $\mathrm{C}=\mathrm{C}$ stretching) and $1,265 \mathrm{~cm}^{-1}(\mathrm{C}=\mathrm{O}$ stretching $)$. Based on the FTIR results, it was revealed that the microcapsules contained the chemical ingredients and functional groups of the wall material (agarose and gelatin) and the core active agent (gallic acid). The overall results confirm the synthesis of the microcapsules containing gallic acid.

In vitro release of microcapsules. Fig. 7 illustrates the release profile of the microcapsules containing gallic acid. The percentage of drug release was investigated for $96 \mathrm{~h}$ in the in vitro controlled release model. Gallic acid was released gradually in the phosphate $(\mathrm{pH}$ 7.4) medium. After the first $6 \mathrm{~h}$, it was observed that $>15 \%$ of the drug was released from the microcapsules. After $24 \mathrm{~h}$, approximately $40 \%$ of the drug was released. Another $13 \%$ of gallic acid was released after $48 \mathrm{~h}$, and the release a 72 and $96 \mathrm{~h}$ was close to the plateau level. 
Table II. Zone of clearance of agarose/gelatin-based microcapsules containing gallic acid towards A. niger.

Zones of clearance $(\mathrm{mm})$

\begin{tabular}{lcccr}
\cline { 3 - 5 } Dose & Gallic acid & $\begin{array}{c}\text { Microcapsules containing } \\
\text { gallic acid }\end{array}$ & $\begin{array}{c}\text { Blank } \\
\text { microcapsules }\end{array}$ & Terbinafine \\
\hline $250 \mu \mathrm{g}$ & Nil & $2.87 \pm 0.51$ & Nil & \\
$500 \mu \mathrm{g}$ & Nil & $6.37 \pm 0.64$ & Nil & $19.33 \pm 0.93$ \\
$1,000 \mu \mathrm{g}$ & Nil & $9.17 \pm 0.35$ & Nil &
\end{tabular}

Results are shown as the means \pm SD. A. niger, Aspergillus niger; SD, standard deviation.

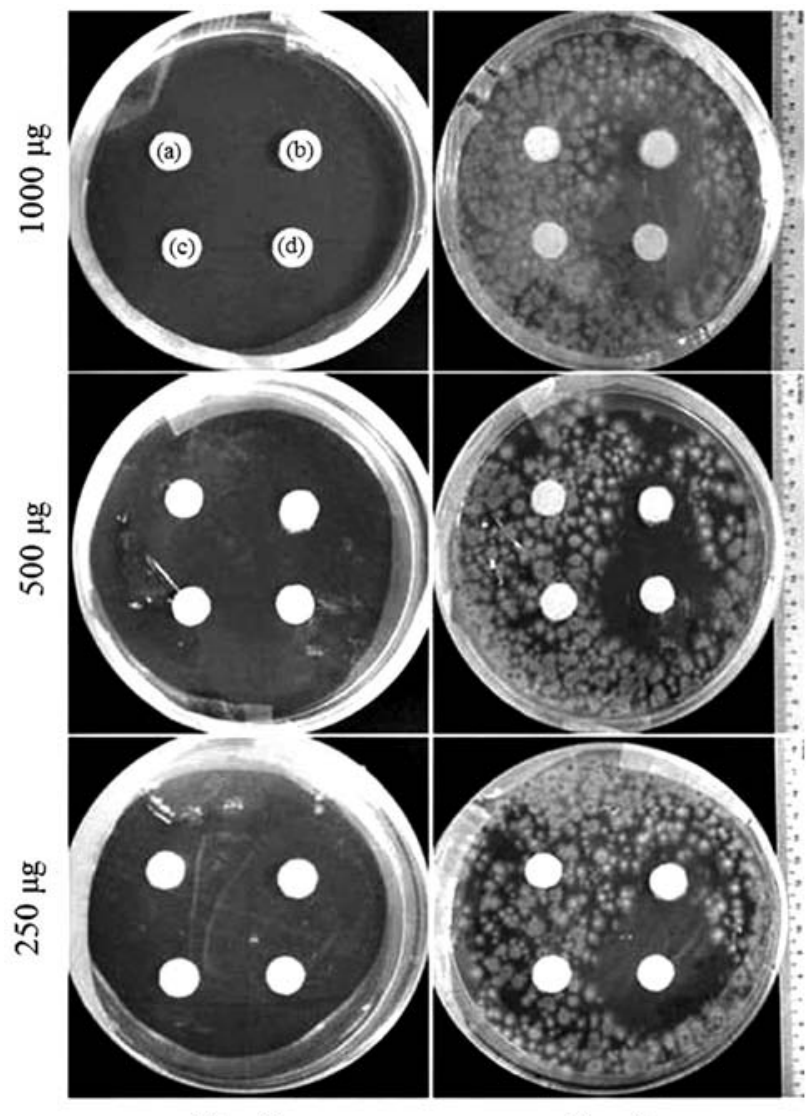

Day 0

Day 2

Figure 8. Growth inhibitory effects towards Aspergillus niger: (A) free gallic acid; (B) microcapsules containing gallic acid; (C) blank microcapsules (negative control); and (D) $5 \mu \mathrm{g}$ terbinafine (positive control).

Determination of MICs of microcapsules. The MICs demonstrated the lowest concentration of the microcapsules containing gallic acid and gallic acid allowing no growth of A. niger as shown in Table I. Terbinafine was used as a positive control. It was observed that the MIC of microencapsulated gallic acid $(62.5 \mu \mathrm{g} / \mathrm{ml})$ was greatly improved when compared to that of free gallic acid $(>750 \mu \mathrm{g} / \mathrm{ml})$. This may be due to the fact that the fungi normally take $48 \mathrm{~h}$ to grow and the agarose/gelatin wall matrix offered a relatively stable and sustained release of gallic acid. This may result in a continuous inhibitory effect of microencapsulated gallic acid towards A. niger.
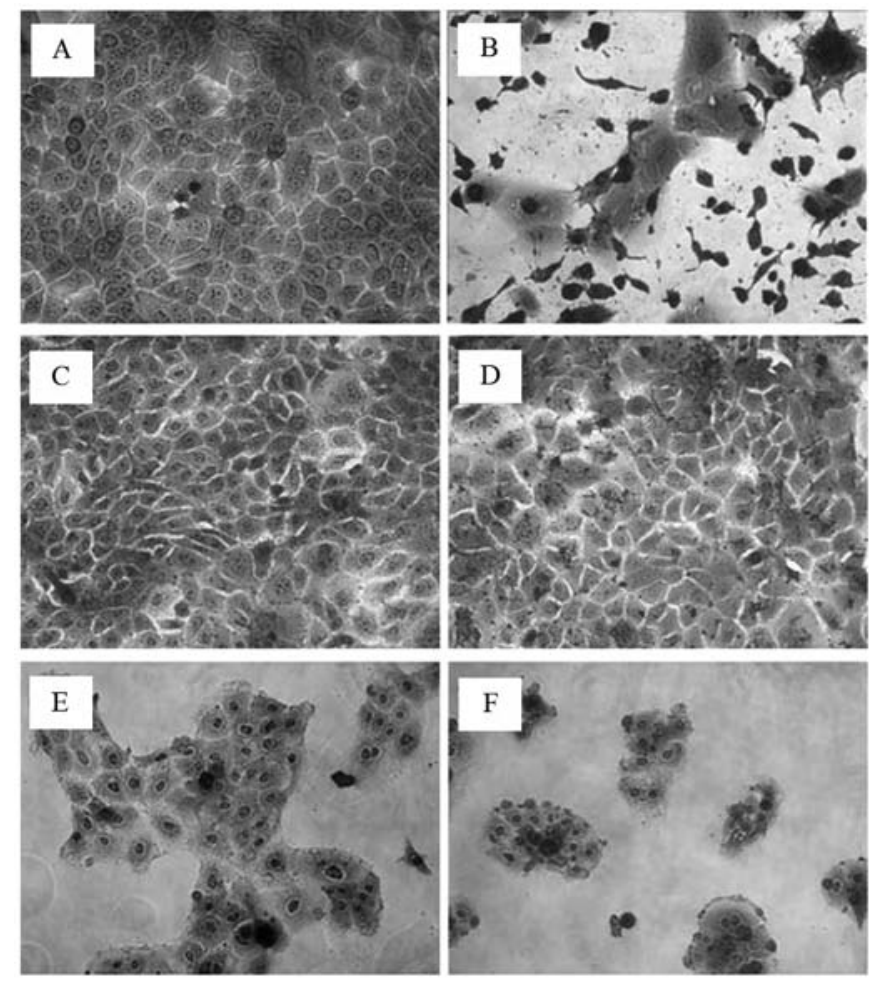

Figure 9. Inhibitory effects towards human skin keratinocytes: (A) blank control microcapsules; (B) $2 \mu \mathrm{g} / \mathrm{ml}$ doxorubicin; (C) microcapsules containing $62.5 \mu \mathrm{g} / \mathrm{ml}$ gallic acid; (D) microcapsules containing $125 \mu \mathrm{g} / \mathrm{ml}$ gallic acid; (E) $62.5 \mu \mathrm{g} / \mathrm{ml}$ gallic acid; and (F) $125 \mu \mathrm{g} / \mathrm{ml}$ gallic acid.

Antifungal activity of microcapsules. Fig. 8 and Table II show the dose-dependent response of microencapsulated gallic acid to A. niger activity. Microcapsules containing $1,000 \mu \mathrm{g}$ of gallic acid (inhibitory zone, $9.17 \pm 0.35 \mathrm{~mm}$ ) had the strongest anti-A. niger activity, followed by microcapsules with $500 \mu \mathrm{g}$ (inhibitory zone, $6.37 \pm 0.64 \mathrm{~mm}$ ) and $250 \mu \mathrm{g}$ (inhibitory zone, $2.87 \pm 0.51 \mathrm{~mm}$ ) of gallic acid. However, free gallic acid did not show any growth inhibitory effects towards A. niger, similar to the blank microcapsules (negative control). It was speculated that the free drug may reduce the fungal growth at the beginning. The noticeably stronger antifungal activity of microencapsuleated gallic acid may be due to the prolonged drug release from the microcapsules which provided a continuous inhibitory effect towards the fungal cells which grew relatively slow within $48 \mathrm{~h}$. 


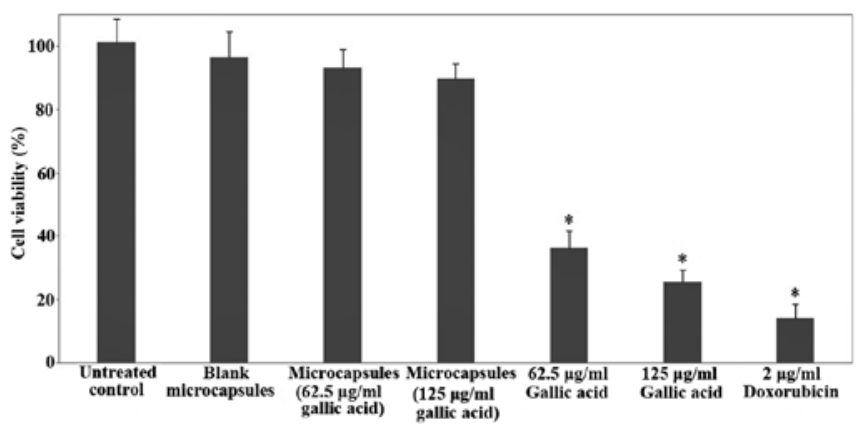

Figure 10. Effects of microcapsules containing gallic acid on the viability of human skin keratinocytes. Three independent experiments were performed in triplicate and similar results were obtained. The results are presented as the means \pm standard deviation ( $\mathrm{p}$-values $<0.05$ ).

Cytotoxicity of microcapsules. As shown in Fig. 9, it was observed that the microcapsules containing gallic acid at a dose of $62.5 \mu \mathrm{g} / \mathrm{ml}$ (Fig. 9C) and $125 \mu \mathrm{g} / \mathrm{ml}$ (Fig. 9D) did not induce cell death. Morphologically, microcapsules containing gallic acid at a dose if $62.5 \mu \mathrm{g} / \mathrm{ml}$ (Fig. 9C) and $125 \mu \mathrm{g} / \mathrm{ml}$ (Fig. 9D) also showed a normal cellular morphology, similar to the blank control microcapsules (Fig. 9A). The microcapsules containing gallic acid also exhibited a similar effect on cell viability (Fig. 10) to the untreated controls and blank control microcapsules; cell survival was $>90 \%$ following treatment with microcapsules containing gallic acid at $62.5 \mu \mathrm{g} / \mathrm{ml}$ and approximately $90 \%$ following treatment with microcapsules containing gallic acid at $125 \mu \mathrm{g} / \mathrm{ml}$. Free gallic acid at the concentration of $62.5 \mu \mathrm{g} / \mathrm{ml}$ (Fig. 9E) and $125 \mu \mathrm{g} / \mathrm{ml}$ (Fig. 9F), however, induced significant cell death, indicated by nuclear condensation, cell shrinkage and detachment from the substratum. As shown in Fig. 10, cell viability following treatment with free gallic acid at 62.5 and $125 \mu \mathrm{g} / \mathrm{ml}$ was approximately $35 \%$ and $25 \%$, respectively when compared with the untreated controls. Doxorubicin at $2 \mu \mathrm{g} / \mathrm{ml}$ (Fig. 9B) was used as a positive control to induce HaCaT skin keratinocyte cell death. The blank control microcapsules did not inhibit cell proliferation or induce cell death.

Polymeric microcapsules have been widely applied for drug delivery in order to improve the therapeutic performance of original drugs. Cutaneous aspergillosis is generally caused by Aspergillus fungi and it can be associated with A. niger, which leads to skin infections at or near intravenous injection sites. In order to utilize the biological effects of therapeutic agents, microencapsulation is considered a feasible method since the concentration of microencapsulated drugs in the targeted body sites can be modulated in a controlled manner. The drug bioavailability can also be improved with the aid of the microparticle delivery system, as microencapsulated drugs increase the stability, safety and efficacy of the original drugs, and therefore improve the therapeutic effects of drug treatment and promote patient compliance and convenience of administration. Recently, we reported the use of an agar/gelatin-based microencapsulation system to deliver gallic acid for hepatoprotective application (5) and to deliver Phyllanthus urinaria for antifungal purposes (15). In order to enhance the potential antifungal activity of gallic acid in the microencapsulation system, agarose was used instead of agar in the gelatinbased system due to the stronger mechanical properties of the agarose gel network. The development, characterization and biological activities of the agarose/gelatin-based microcapsules containing gallic acid were demonstrated in this study. The involvement of agarose as the wall matrix materials with gelatin aimed at strengthening the wall of the formed microcapsules and avoiding the potential toxicity of using the hazardous crosslinking agent, glutaraldehyde. The development of an optimal microencapsulation recipe without the use of glutaraldehyde may promote the desired properties of microcapsules due to its biological safety and economic advantages in microcapsule formation. Our results revealed that microencapsulated gallic acid markedly improved the antifungal activity towards $A$. niger, but also greatly reduced the cytotoxic effects towards human skin keratinocytes when compared with the original drug. Therefore, it was speculated that the development of biologically safe agarose/gelatin-based microcapsules containing gallic acid may be a potent device for the treatment of cutaneous aspergillosis.

\section{Acknowledgements}

This study was supported by the Innovative Technology Commission, HKPU, and the accounts of 38-40-116 (C.H.C.) and 03-16-176 (X.Z.B.). We also acknowledge the financial support from the research fund of Ministry of Education (MOE) Key Laboratory for Regenerative Medicine, School of Biomedical Sciences, Chinese University of Hong Kong (K.K.-H.L).

\section{References}

1. Stevens DA, Kan VL, Judson MA, Morrison VA, Dummer S, Denning DW, Bennett JE, Walsh TJ, Patterson TF and Pankey GA: Practice guidelines for diseases caused by Aspergillus. Infectious Diseases Society of America. Clin Infect Dis 30: 696-709, 2000.

2. van Burik JA-H, Colven R and Spach DH: Cutaneous aspergillosis. J Clin Microbiol 36: 3115-3121, 1998.

3. Pal AK, Gajjar DU and Vasavada AR: DOPA and DHN pathway orchestrate melanin synthesis in Aspergillus species. Med Mycol 1: 10-18, 2014

4. Baker SE: Aspergillus niger genomics: past, present and into the future. Med Mycol 44: S17-21, 2006.

5. Lam PL, Lee KKH, Kok SHL, Cheng GYM, Tao XM, Hau DKP, Yuen MCW, Lam KH, Gambari R, Chui CH and Wong RSM: Development of formaldehyde-free agar/gelatin microcapsules containing berberine $\mathrm{HCl}$ and gallic acid and their topical and oral applications. Soft Matter 8: 5027-5037, 2012.

6. Ma J, DU LF, Chen M, Wang HH, Xing LX, Jing LF and Li YH: Drug-loaded nano-microcapsules delivery system mediated by ultrasound-targeted microbubble destruction: A promising therapy method. Biomed Rep 1: 506-510, 2013.

7. Lam PL, Kok SH, Bian ZX, Lam KH, Tang JC, Lee KK, Gambari R and Chui $\mathrm{CH}$ : D-glucose as a modifying agent in gelatin/collagen matrix and reservoir nanoparticles for Calendula officinalis delivery. Colloids Surf B Biointerfaces 117: 227-283, 2014.

8. Choubey $\mathrm{J}$ and Bajpai AK: Investigation on magnetically controlled delivery of doxorubicin from superparamagnetic nanocarriers of gelatin crosslinked with genipin. J Mater Sci: Mater Med 21: 1573-1586, 2010.

9. Migneault I, Dartiguenave C, Bertrand MJ and Waldron KC: Glutaraldehyde: behavior in aqueous solution, reaction with proteins, and application to enzyme crosslinking. Biotechniques 37: 790-796, 798-802, 2004.

10. Zeiger E, Gollapudi B and Spencer P: Genetic toxicity and carcinogenicity studies of glutaraldehyde - a review. Mutat Res 589: $136-151,2005$. 
11. Takigawa $\mathrm{T}$ and Endo Y: Effects of glutaraldehyde exposure on human health. J Occup Health 48: 75-87, 2006

12. Corrado OJ, Osman J and Davies RJ: Asthma and rhinitis after exposure to glutaraldehyde in endoscopy units. Human Toxicology 5: 325-328, 1986.

13. Pisaniello DL, Gun RT, Tkaczuk MN, Nitshcke M and Crea J: Glutaraldehyde exposures and symptoms among endoscopy nurses in South Australia. Appl Occup Environ Hyg 12: 171-177, 1997.

14. Stern ML, Holsapple MP, McCay JA and Munson AE: Contact hypersensitivity response to glutaraldehyde in guinea pigs and mice. Toxicol Ind Health 5: 31-43, 1989.

15. Lam PL, Kok SH, Ho YW, Wong RS, Cheng GY, Cheng CH, Lam KH, Gambari R, Lee KK and Chui CH: A novel green gelatin-agar microencapsulation system with $P$. urinaria as an improved anti-A. niger model. Carbohydr Polym 92: 877-880, 2013.

16. Bao X, Hayashi K, Li Y, Teramoto A and Abe K: Novel agarose and agar fibers: fabrication and characterization. Mater Lett 64 2435-2437, 2010

17. Zhou QZ, Wang LY, Ma GH and Su ZG: Multi-stage premix membrane emulsification for preparation of agarose microbeads with uniform size. J Membr Sci 322: 98-104, 2008.

18. Almrhag O, George P, Bannikova A, Katopo L, Chaudhary D and Kasapis S: Investigation on the phase behaviour of gelatin/ agarose mixture in an environment of reduced solvent quality. Food Chem 136: 835-842, 2013.

19. Clark LJ, Whalley WR, Leigh RA, Dexter AR and Barraclough PB: Evaluation of agar and agarose gels for studying mechanical impedance in rice roots. Plant Soil 207: 37-43, 1999

20. Sakai S, Hashimoto I and Kawakami K: Agarose-gelatin conjugate membrane enhances proliferation of adherent cells enclosed in hollow-core microcapsules. J Biomater Sci Polymer Ed 19: 937-944, 2008

21. Park W, Chang MS, Kim H, Choi HY, Yang WM, Kim do R, Park EH and Park SK: Cytotoxic effect of gallic acid on testicular cell lines with increasing $\mathrm{H}_{2} \mathrm{O}_{2}$ level in GC-1 spg cells. Toxicol In Vitro 22: 159-163, 2008
22. Akiyama H, Fujii K, Yamasaki O, Oono T and Iwatsuki K: Antibacterial action of several tannins against Staphylococcus aureus. J Antimicrob Chemother 48: 487-491, 2001.

23. Hussin NM, Muse R, Ahmad S, Ramli J, Mahmood M, Sulaiman MR, Shukor MYA, Rahman MFA and Aziz KNK: Antifungal activity of extracts and phenolic compounds from Barringtonia racemosa L. (Lecythidaceae). Afr J Biotechnol 8: 2835-2842, 2009.

24. Li L, Ng TB, Gao W, Li W, Fu M, Niu SM, Zhao L, Chen RR and Liu F: Antioxidant activity of gallic acid from rose flowers in senescence accelerated mice. Life Sci 77: 230-240, 2005.

25. Zhao B and $\mathrm{Hu} \mathrm{M}$ : Gallic acid reduces cell viability, proliferation, invasion and angiogenesis in human cervical cancer cells. Oncol Lett 6: 1749-1755, 2013.

26. Liang W, Li X, Li Y, Li C, Gao B, Gan H, Li S, Shen J, Kang J, Ding S, Lin X and Liao L: Gallic acid induces apoptosis and inhibits cell migration by upregulating miR-518b in SW1353 human chondrosarcoma cells. Int J Oncol 44: 91-98, 2014

27. Yang YM and Park WH: p38 inhibitor enhances growth inhibition and death in gallic acid-treated endothelial cells. Int J Mol Med 26: 295-300, 2010.

28. Niho N, Shibutani M, Tamura T, Toyoda K, Uneyama C, Takahashi N and Hirose M: Subchronic toxicity study of gallic acid by oral administration in F344 rats. Food Chem Toxicol 39: 1063-1070, 2001

29. Lam PL, Lu GL, Hon KM, Lee KW, Ho CL, Wang X, Tang JC, Lam KH, Wong RSM, Kok SH, Bian ZX, Li H, Lee KKH, Gambari R, Chui $\mathrm{CH}$ and Wong WY: Development of ruthenium(II) complexes as topical antibiotics against methicillin resistant Staphylococcus aureus. Dalton Trans 43: 3949-3957, 2014.

30. Oza MD, Prasad K and Siddhanta AK: One-pot synthesis of fluorescent polysaccharides: adenine grafted agarose and carrageenan. Carbohydr Res 357: 23-31, 2012. 